\title{
Genome-wide Analysis Reveals Class and Gene Specific Codon Usage Adaptation in Avian Paramyxoviruses 1
}

Tonya L. Taylor, Kiril M. Dimitrov, and Claudio L. Afonso* 


\section{ABSTRACT}

In order to characterize the evolutionary adaptations of avian paramyxovirus 1 (APMV1) genomes, we have compared codon usage and codon adaptation indexes among groups of Newcastle disease viruses that differ in biological, ecological, and genetic characteristics. We have used available GenBank complete genome sequences, and compared codon usage of class I (CI-29 sequences containing 132,675 codons) and class II (CII-259 sequences containing 1,184,925 codons) APMV-1 genomes. We also compared available complete fusion protein gene sequences (CI-175 sequences containing 96,775 codons; CII-1166 sequences containing 644,798 codons). Adaptation to Gallus gallus was compared among the different classes of viruses, among different genomic regions based on transcriptional levels, or on the fusion gene.

Interestingly, distinctive codon usage determined by differences in relative synonymous codon usage and by codon adaptation indexes was observed for the two APMV-1 classes and for different transcriptional regions within classes. Furthermore, differential use of the third codon position and preferential use of codon pairs were seen for the two different classes and for selected genotypes of class II despite the fact that there were no large differences in nucleotide composition. The data suggest that codon usage has changed significantly since the two APMV1 classes diverged, however, these changes are not significantly pronounced among viruses of the same genotype, suggesting that codon adaptation in APMV-1 occurs through a slow evolutionary process.

Keywords: APMV-1 classes, RSCU, class II Genotypes, CAI, fusion gene 


\section{Introduction}

Remarkably adaptable viruses, the avian paramyxoviruses of serotype 1 (APMV-1) are capable of infecting over 200 avian species and cause a diverse range of clinical outcomes in birds (Kaleta and Baldauf, 1988). APMV-1 is synonymous with Newcastle disease virus (NDV), with the virulent forms of the virus causing Newcastle disease (Afonso and Miller, 2013). They belong to the genus Avulavirus of the family Paramyxoviridae (Mayo, 2002), are endemic in specific geographic locations, and have also been identified in wild birds worldwide (Dimitrov et al., 2016). The genome of APMV-1 is a single-stranded, negative-sense, non-segmented RNA molecule with the following 6 genes arranged in this $3^{\prime}$ to $5^{\prime}$ order: the nucleocapsid (NC), phosphoprotein $(\mathrm{P})$, matrix $(\mathrm{M})$, fusion $(\mathrm{F})$, hemagglutinin-neuraminidase $(\mathrm{HN})$, and RNA polymerase (L) (Alexander et al., 2012). Although these six transcriptional units all contain their end (Collins et al., 1978; Wilde et al., 1986). genomic RNA and makes up the core structure of the nucleocapsid (Buchholz et al., 1994;

54 Collins et al., 1982). The phosphoprotein binds the ribonucleocapsid, where it positions and 55 stabilizes the RNA polymerase (Buchholz et al., 1994; Hamaguchi et al., 1983). The matrix 56 protein stabilizes the entire virus structure by linking the nucleocapsid to the two important, 57 functional glycoproteins (HN and F) on the surface of the viral envelope (Coronel et al., 2001). 58 The HN protein plays an important role in viral infection through recognizing and attaching to 
host red blood cells, which allows the cleaved F protein, to fuse the virus to the host cell or host

60 cell to host cell, where the viral transcription unit can enter (Huang et al., 2004).These two viral

61 envelope projections have been shown necessary for infection, and are the neutralizing and

62 protective antigens of NDV (Kim et al., 2011; Nagai et al., 1976). However, it is the amino acid

63 sequence at the fusion protein cleavage site that is believed to be the major determinant of

64 virulence (Glickman et al., 1988; Kim et al., 2011).

of only one genotype (CI-G1); whereas, class II contains at least 18 different genotypes (CII-GI-

67 XVIII) (Courtney et al., 2013; Diel et al., 2012b; Snoeck et al., 2013). While certain groups of

68 viruses are preferentially maintained in specific host species, the majority of the virulent

69 genotypes are capable of infecting in chickens (Dimitrov et al., 2016). Viruses of class I are

70 predominantly reported in wild aquatic birds (Kim et al., 2007), and except genotypes I, II and

$71 \mathrm{X}$, the virulent viruses of all other class II genotypes are preferentially isolated from chickens.

72 Class II genotypes VI and VII are responsible for the majority of the most recent outbreaks in

73 poultry; viruses of class II genotype VI are preferentially isolated from Columbiformes (Dimitrov

74 et al., 2016; Dortmans et al., 2010).

Patterns of codon usage normally vary among animal species, and because these species

76 are hosts to viruses, it is expected that viruses would adapt to the translational codon usage of

77 different host organisms by changing its codon usage to achieve maximum fitness (Bahir et al.,

78 2009; Knight et al., 2001). However, other factors such as nucleotide compositional bias,

79 transfer RNA (tRNA) abundance, RNA stability and expression levels have been found to be

80 responsible for non-random codon usage (Knight et al., 2001). One form of non-random codon

81 usage is the suboptimal use of codons to negatively regulate expression of genes for which 
82 excessive expression decreases fitness (Coleman et al., 2008). Here, we have used the Gallus

83 gallus codon dataset available in the Data Analysis in Molecular Biology and Evolution

84 (DAMBE v5.5.2) software package to search for evidence of differential host adaptation and

85 codon usage in APMV-1 (Xia, 2013). We have compared the coding sequences of the fusion gene (CI-175 sequences containing 96,775 codons; CII-1166 sequences containing 644,798

87 codons) and the coding sequences of complete genomes (CI-29 sequences containing 132,675 codons and CII-259 sequences containing 1,184,925 codons).

Previously, synonymous codon usage of the fusion protein gene (Kumar and Kumar,

90 2014) and the complete genome (Wang et al., 2011) in NDV was analyzed, and both studies

91 found only random mutational pressure and a low amount of codon usage bias. In the light of

92 those results, here, we have performed genome wide and gene specific comparisons of codon

93 usage, focusing mainly on two distinct groups of viruses: viruses of low virulence of class I that

94 are known to be maintained in waterfowl and viruses of class II, the majority of which are

95 virulent. Among virulent viruses, we have compared viruses normally expected to be maintained

96 in chickens (genotype VII) versus viruses that are normally maintained in pigeons (genotype VI).

97 In addition, we have determined the relative synonymous codon usage (RSCU), the codon

98 adaptation index (CAI) to Gallus gallus, the effective number of codons, the nucleotide

99 composition and the di-codon frequency, and compared these analyses among class I and class II

100 viruses. Our data demonstrates differences in codon usage within the fusion gene and in the full

101 genome between class I and class II viruses, and the differences in codon bias in different

102 genotypes of class II. This work also provides further investigation into the relationship between

103 levels of APMV-1 gene expression and codon usage. 


\section{Materials and methods}

1052.1 Sequence data

Complete genome sequences of 330 APMV-1 isolates were downloaded from GenBank

107 (http://www.ncbi.nlm.nih.gov/nuccore; as of June 20 2016 ); in addition, 1,819 sequences of the

108 complete coding region of the fusion protein gene were also available and downloaded.

109 Duplicate and recombinant sequences, sequences of man-made clone viruses and sequences that

110 were obtained from vaccine-like viruses were removed from the datasets in order to analyze only

111 viruses that represent natural evolutionary and adaptation processes. Some additional sequences

112 for which background data was missing were also removed. As a result, 288 complete genome

113 sequences and 1,341 fusion gene sequences were used for subsequent analyses. The coding

114 sequences for both the complete genome and the fusion gene were grouped into datasets

115 according to class and genotype using the criteria described by Diel et al. (Diel et al., 2012b).

116 The accession numbers, as well as location, year, and host of isolation are also provided in the

117 Supplementary materials (Tables S1 and S2). To look at differentially expressed genomic

118 regions, the APMV-1 genome was divided into 3 regions. The regions were conditionally named

119 as follows: nucleocapsid-phosphoprotein-matrix (NC-PHO-M), fusion-haemagglutinin-

120 neuraminidase (F-HN), and polymerase (POL). All coding sequences were aligned using the

121 MAFFT v7.222 alignment tool as implemented in Geneious v9.1.5 (Katoh et al., 2002).

122 2.2. Calculation of relative synonymous codon usage (RSCU) and codon adaptation index (CAI)

123 Relative synonymous codon usage was calculated for each sequence, as previously

124 described (Sharp and Li, 1986). Equation 1 below was used as implemented in DAMBE v5.5.2

125 software package (Xia, 2013). 


$$
R S C U=\frac{X_{i j}}{\Sigma_{j}^{n i} X_{i j}} n_{i}
$$

127 RSCU is the value of the observed codon number $(\mathrm{X})$ divided by the expected codon number

$128(\Sigma)$; the expected codon number assumes an occurrence where the codons (i) for the same amino 129 acid (j) were all used equally ( $n_{i}$ is the number of synonymous codons). RSCU values of greater 130 than 1 have a positive codon usage bias and were considered abundantly used codons. Those that 131 have RSCU values less than 1 have a negative codon usage bias and were used less. Codons with 132 RSCU values of 1 lack any bias and were used equally within the genome or that particular 133 genomic region.

In order to study the preference of codon usage in APMV-1, we used the codon

135 adaptation index calculated using DAMBE (Sharp and Li, 1987; Xia, 2013). Based on the coding 136 sequences, this method predicts expression levels, and compares the codon usage of the different 137 APMV-1 isolates to a predetermined reference set, in this particular case, Gallus gallus. All 1381,341 fusion gene sequences were used to analyze the adaptation of class I and class II viruses to 139 Gallus gallus. In addition, the 288 complete genome sequences and each of the 3 designated 140 genomic regions (NC-PHO-M, F-HN, and POL) were used to determine the CAI value to Gallus 141 gallus and to compare between the two classes. Codons with RSCU values of 1 and stop codons 142 were excluded from the analysis.

145 regions (NC-PHO-M, F-HN, and POL), and the complete genome for both class I and class II 146 viruses, using the GraphPad Prism 6 software, to determine the linear relationship among the 147 samples ( $\mathrm{r}$ ). The $\mathrm{r}$ coefficient was used to determine the presence and magnitude of the 
148 correlation, and the two-tailed $\mathrm{P}$ test $(\mathrm{P}<0.01)$ was used to determine significance. Correlation 149 plots with a Pearson r coefficient of 0.5 to 1 have a high correlation, 0.3 to 0.5 have a medium 150 correlation, 0.1 to 0.3 have low correlation, and less than 0.1 have no correlation. In addition, 151 linear regression analysis was also performed to support correlation among the different samples.

152 Those samples with $\mathrm{R}^{2}$ values close to 0 indicates no relationship between the two variables, and 153 the closer the $\mathrm{R}^{2}$ value gets to 1 , the stronger the relationship between the two variables.

154 2.4. Nucleotide composition and di-codon frequency

Class I $(n=175)$ and class II $(n=1,166)$ fusion gene coding sequences and class $I(n=29)$

156 and class II ( $\mathrm{n}=259)$ complete genome coding sequences were submitted to DAMBE for

157 nucleotide composition analysis. For both class I and class II fusion gene sequences, 1,659

158 nucleotides were analyzed with 553 nucleotides for each of the three positions, and for the 159 complete genome of both classes, 13,725 nucleotides were analyzed with 4,575 nucleotides for 160 each of the three positions. Values were given in percent with the total nucleotide count divided 161 by the total count of each specific nucleotide at each of the three positions. In addition, the total 162 GC content and GC content for the $3^{\text {rd }}$ codon position (GC3) for each sequence were also 163 determined by DAMBE.

Di-codon frequency was also determined using the DAMBE software employing all 1651,341 fusion gene and 288 complete genome sequences. The observed count for each codon pair 166 (4,096 pairs total) was divided by the expected counts to determine the di-codon frequency given 167 in percentage. To obtain the expected counts, the total of codon pairs that encode the same set of 168 amino acids was divided by the value given when the codon degeneracy of each of the amino 
169 acids in the set was multiplied. This value assumes that each codon pair codes for the amino acid 170 set equally.

$171 \quad 2.5$. Effective Number of Codons (ENC)

The effective number of codons for the fusion gene and complete genome of each

173 sequence was calculated using Equation 2 as implemented in DAMBE.

174

$$
E N C=2+\mathrm{s}+\{29 /[\mathrm{s} 2+(1-\mathrm{s} 2)]\}
$$

ENC values range from 20 to 61, with 20 being exceptionally rare, indicating only one

176 synonymous codon for each amino acid was used, where an ENC value of 61 corresponds to the

177 use of all synonymous codons equally (Wright, 1990). The s in Equation 2 equals GC3. The

178 average ENC and GC3 values of all sequences were calculated, and the average of each class and

179 genotype were used to create ENC vs GC3 scatter plots. In addition, the average ENC and CAI

180 value of all of the sequences were calculated, and the average of each class and genotype were

181 used to create CAI vs ENC plots. The $\mathrm{r}$ coefficient was used to determine the presence and

182 magnitude of the correlation, and the two-tailed $\mathrm{P}$ test $(\mathrm{P}<0.01)$ was used to determine

183 significance. Linear regression analysis was also performed to support correlation among the 184 different samples, with a 95\% confidence level, and the line was constrained to go through the 185 origin.

$186 \quad 2.6$ Statistical Analysis

The 2-way analysis of variance (ANOVA) method, with Dunn-Sidak's multiple test correction was used to perform statistical analysis on the different experimental results through the GaphPad Prism 6 software with significance defined as $\mathrm{P}<0.01$. 
Bayesian time scaled analyses were conducted by the Bayesian Markov Chain Monte

192 Carlo (BMCMC) method implemented in BEAST v1.8.2 (Drummond et al., 2012) program utilizing a polymerase gene sequence dataset. The polymerase gene was selected for molecular

194 clock estimations because, as an internal gene, it is expected to be less influenced by selective 195 pressure and is known to have a high level of genetic conservation (Kim et al., 2008; Fuller et al., 196 2010). The L-gene also occupies almost half of the APMV-1 genome. The used dataset was 197 parsed from the available complete genome sequences (Supplemental Table S2). Sequences from 198 the same year and genotype were removed, resulting in a final dataset of 155 full L-gene 199 sequences isolated between 1933 and 2014. General time reversible and Hasegawa, Kishino, and 200 Yano with gamma distribution nucleotide substitution models were applied separately for 201 comparison (GTR $+\Gamma_{4}$ and $\mathrm{HKY}+\Gamma_{4}$, respectively). Relaxed clock model (uncorrelated lognormal 202 distribution) was tested with different demographic models (nonparametric Bayesian skyline 203 plot, parametric exponential growth and constant coalescent size), and the best model was 204 selected based on marginal likelihood estimations using path sampling/stepping-stone sampling 205 (Baele et al., 2012). An input file for BEAST analysis was prepared using Bayesian evolutionary 206 analysis utility software v.1.8.2, in which sequences were annotated with year of collection.

207 Three independent chains were run to get output of 10,000 trees. Convergence was assessed in 208 Tracer v1.6.0 program (http://beast.bio.ed.ac.uk/Tracer). Uncertainties in BMCMC estimates 209 were reflected by the ranges of the 95\% highest posterior density (95\% HPD). Maximum clade 210 credibility trees were generated using the Tree Annotator program from the BEAST package, 211 and the FigTree program v1.4.2 (http://tree.bio.ed.ac.uk/software/figtree) was used for the 212 visualization of the annotated trees. 


\section{3. Results}

214 3.1. Differences in the full fusion coding sequence RSCU between class I and class II viruses

215 After we removed recombinant viruses and viruses that were apparent vaccines and vaccine

216 escapes, 1,341 available sequences were used to extend our study across class I (175) and class II

217 (1,166) viruses (Supplemental Table S1) to study the fusion gene RSCU. RSCU is defined as the

218 number of times a codon appears divided by the number of expected occurrences when all

219 codons are equally used. RSCU values were determined for each sequence from both APMV-1

220 classes, and the averages for each class are reported in Supplemental Table S3. Values of RSCU

221 greater than 1 indicate more frequent use of that codon than expected; whereas, values of RSCU

222 less than 1 indicate use of that codon less than expected. Figure 1A shows only the average

223 RSCU values of the fusion gene for the codon usage that was found to be statistically different,

224 using 2-way ANOVA, between the class I and class II viruses (bold font in Table S3), and clear

225 differences in average RSCU values were seen in multiple codons. In addition, our analysis led

226 us to question whether there were any codons whose usage was statistically different between

227 class I and class II viruses in the fusion gene that were not found in the complete genome, and

228 fifteen codons were discovered to be differentially used between class I and class II viruses only

229 in the fusion gene (boxed values in Table S3).

To study whether the fusion gene provided a good representation of the differences of

231 codon usage between class I and class II viruses, a Pearson Correlation plot was made to

232 visualize the relationship between class I and class II RSCU (Fig. 1B). Correlation plots with a

233 Pearson $\mathrm{r}$ coefficient of 0.5 to 1 have a high correlation, 0.3 to 0.5 have a medium correlation,

2340.1 to 0.3 have low correlation, and less than 0.1 have no correlation. A Pearson $r$ coefficient of

2350.35 was observed when analyzing the RSCU values of class II versus class I sequences, 
236 indicating a weak relationship among codon usage between the two classes of viruses. This weak

237 relationship was due to the statistically significant differences in codon usage between class I and

238 class II viruses (Table S3). In contrast, when class II sequences were compared against other

239 class II sequences, a high, positive correlation is displayed with $\mathrm{r}$ coefficients of 0.8 (data not

240 shown).

241 We wanted to determine if adaptive pressures have affected the differences in codon

242 usage of the fusion gene between the two classes of viruses and among the genotypes of class II.

243 ENC measures codon usage bias, but this measure does not distinguish codon usage bias that was

244 due to A/T bias or GC3 content (Vicario et al., 2007). The relationship between ENC and GC3

245 can be used to decipher between codon usage bias that was due to A/T bias or preferred GC

246 content at the wobble position. For that purpose, the ENC and GC3 values for the fusion gene of

247 all sequences were also calculated and used to create a scatter plot for each class and genotype

248 (Fig 1C). The average ENC value was 56 for both classes. Average GC3 values ranged from

24943.3 to 50.1, with class I having the highest GC3 value, indicating an evolutionary decrease in

250 the use of GC at position 3 in class II sequences.

251 To understand whether gene expression was correlated with codon usage, CAI vs ENC

252 scatter plots were created (Figs. 1D and 1E). Whereas ENC is a non-directional measure that

253 observes the unequal use of codons across the coding regions, CAI is a directional measure of

254 codon usage, and will explain deviation from the expected codon usage in comparison to the

255 codon usage of a reference genome (Vicario et al., 2007). The ENC and CAI values for the

256 fusion gene of all sequences were calculated and plotted. Class I has a negative correlation with

257 an r value of -0.8983 (Fig. 1D). Class II (Fig. 1E) has a loss of correlation between CAI and

258 ENC with an $\mathrm{r}$ value very close to zero (-0.09976). 
To further compare codon usage in the fusion gene between class I and the class II

260 viruses, we analyzed di-codon frequency, and discovered major differences between class I and

261 class II sequences, as well as differences between class II genotypes VI and VII (Table S4). A di-

262 codon is six consecutive nucleotides in a row, and there are 4,096 possible di-codon pairs. Di-

263 codon frequency, the di-codon pairs that were observed versus the number that should be

264 expected, can be used to indicate further codon usage bias. The 35 di-codon pairs with the

265 highest frequency within the fusion gene across class I and class II sequences are represented in

266 Supplemental Table S4.

267 Additionally, we studied the codon usage patterns of the fusion gene in class II sequences

268 based on predicted virulence (deduced amino acid motif of the cleavage site) $(\mathrm{n}=1,341$, number

269 of viruses isolated from 1933 to 2014), location ( $n=59$, number of countries), and geography.

270 However, we did not find any evidence of adaptation within classes or significant differences

271 due to virulence and/or geographic location. No significant difference in codon usage was found

272 between virulent and non-virulent viruses of the same genotype or viruses isolated in different

273 continents (data not shown).

274

275 3.2. Variation in codon adaptation is observed among the three genomic regions of class I and $276 \quad$ class II

277 We divided the genome into three regions (NC-PHO-M, F-HN, and POL) based on

278 transcriptional polarity and studied the codon usage per genomic region in 288 complete

279 genomes. We began by comparing the RSCU within the different genomic regions, and in

280 addition to the variation found within the fusion gene, the existence of additional variation within

281 each region between class I and class II sequences was determined. In all 3 regions, codons that 
282 encode for amino acids G, I, L, P, Q, R, S, T, and V have statistically different RSCU values

283 between class I and class II sequences (Fig S1). Fig S1 represents the statistically significant

284 differences on distribution and bias on the RSCU data from Table 1, which shows that despite all

285 regions utilizing GCA (A), UUC (F), and AAG (K) similarly, the codon usage bias among

286 different regions is evident. In addition, no association between the number of synonymous

287 codons per amino acid and chemical composition was observed (data not shown).

To understand the relationship between the different transcriptional regions and codon

289 usage, we looked at those codons that were statistically different between class I and class II in

290 the three differentially expressed genomic regions (Table 1), since there were minor (not

291 statistically significant) differences between the RSCU values among the regions specifically. In

292 the NC-PHO-M region, there were 38 codons used significantly different between class I and

293 class II (bold in Table 1); the codon usage of five of those codons (UGU, UGC, AUA, CUC, and

294 ACA) was specifically only found to be significant in the NC-PHO-M region (blue in Table 1).

295 In the F-HN region, there were 35 codons used significantly different between class I and class II

296 (bold in Table 1); the codon usage of five of those codons (GAG, GAA, GGC, UCG, and GUA)

297 was specifically only found to be significant in the F-HN region (red in Table 1). In the POL

298 region, there were 40 codons, used significantly different between class I and class II (bold in

299 Table 1); the codon usage of seven of those codons (GCU, GCG, GCC, GAU, GAC, AGC, and

300 AGU) was specifically only found to be significant in the POL region (green in Table 1). It is

301 interesting to note, that AUC (I), CUA (L), AAC (N), AAU (N), CGC (R), UCU (S), ACC (T),

302 UAC (Y) and UAU (Y) were found to be statistically different between class I and class II in

303 both the NC-PHO-M and POL regions but not within the F-HN region. 
To expand our analysis on codon usage bias, the CAI values determined for the class I and

class II sequences were also used to visualize codon adaptation to Gallus gallus in Fig. 2A.

Those sequences with higher CAI values are considered to be more adapted towards the host. CAI values of all three genomic regions were statistically different $(\mathrm{P}<0.01)$ when comparing adaptation between class I and class II viruses to Gallus gallus (corresponding letters in Fig. 2A). In addition, all regions were statistically different within each class; however, the class II F-HN genomic region has a lower CAI value compared to the class II complete genome but the same trend was not observed in class I. Due to this result and to further understand more about how gene expression is correlated with codon usage bias in the F-HN genomic region, we once again determined the CAI (to Gallus gallus) and ENC values for each class I ( $\mathrm{n}=29)$ and class II $(\mathrm{n}=259)$ sequence, and plotted the values in CAI vs ENC scatter plots per class (Figs. 2B-C). The class I F-HN region (Fig. 2B) had a negative r value of -0.9033 , with average ENC values around 56.6 and average CAI values around 0.743. The class II F-HN region (Fig. 2C) had a positive $\mathrm{r}$ value of 0.1828 , with average ENC values around 56.6, and average CAI values around 0.730. The ENC and GC3 value for all sequences in each genomic region and for each class was calculated, and the average for each region and class is plotted in Figure 2D (NC-PHO-M = blue; $\mathrm{F}-\mathrm{HN}=$ red; POL $=$ green). In agreement with the other panels in Figure 2, Figure 2D reiterates that overall class II codon usage is consistently different for class I and II in all regions. The GC3 content of the regions in class II were numerically lower than the class I regions, with the class II F-HN region having the lowest GC3 content among all regions in both classes. The ENC values were very similar (between 56-57) for all regions in both classes, with only a slight difference in the NC-PHO-M regions. Interestingly, the ENC values of the F-HN region of Class I and II, were nearly identical. 
3.3. Codon usage of the complete genome does not match the codon usage of the fusion gene

We have compared the relative synonymous codon usage patterns of the complete

genome between class I and class II sequences. We have analyzed the number and frequencies of codon usage in 288 complete genome datasets (Table S2). Figure 3A displays the average RSCU

332 value of all complete genome sequences of that class that is statistically different between class I 333 and class II viruses, which includes codons that encode amino acids A, F, G, L, N, P, Q, R, S, T 334 and V. Complete genome RSCU values for both class I and class II are found in supplementary 335 Table 3, and those codons in bold indicate the codon usage that is statistically different between class I and class II. Values of RSCU greater than 1 indicate more frequent use of that codon than 337 expected and was found for codons CUA (L), UUG (L), AGG (R) CGC (R), and UAU (Y) in 338 class I only, and UUA (L), CCU (P), AGA (R), CGG (R), ACU (T), and GUC (V) in class II 339 only (Table S3). Significance of differences in codon usage was determined by comparing RSCU 340 values among the numbers of class I and class II sequences using 2-way ANOVA, with Dunn341 Sidaks multiple test correction, with significance defined as $\mathrm{P}<0.01$.

To study whether the complete genome provided a more representative pattern of codon usage between class I and class II viruses, a Pearson Correlation plot was made to visualize the

344 relationship between class I and class II RSCU (Fig. 3B). The average RSCU of all sequences for 345 each class and genotype is represented on the graph. The complete genome of class I and class II 346 viruses show a high, positive correlation with a Pearson $r$ coefficient of 0.84 for each comparison $347 \quad(\mathrm{P}<0.0001)$.

We wanted to determine if the differences seen for the codon usage in the fusion gene of 349 the class II genotypes also existed in the complete genome. Therefore, the ENC and GC3 values 
350 for the complete genome of all sequences were calculated and used to create a scatter plot for

351 each class and genotype in Fig. 3C. The average ENC value was 57-58 for both classes, closer to

352 the maximum 61, the latter indicating use of most synonymous codons. Average GC3 values

353 ranged from 46.6 to 53.7, with class I having the highest GC3 value, indicating an evolutionary

354 decrease in the use of GC at position 3 in class II sequences, in agreement with the fusion gene.

355 The regression line provides a way to associate the effect ENC and GC3 have on each of the 356 groups (Gerdol et al., 2015; Wright, 1990).

We analyzed di-codon frequency to further compare codon usage in the complete genome

358 between class I and class II viruses, including class II genotypes VI and VII, and discovered

359 differences in the di-codons used most often between class I and class II viruses and among class

360 II genotypes (Table S5). The 35 di-codon pairs with the highest frequency within the complete

361 genome across class I and class II sequences are represented in Supplemental Table S5, which

362 are different from the highest frequency di-codon pairs seen in Supplemental Table S4 for the

363 fusion gene.

3.4 Codon usage adaptation in APMV-1 is a slow process.

Because of the small differences in codon usage among different genotypes, we

367 speculated that codon usage adaptation is a slow process that may require long periods of

368 evolution. In order to estimate the time to MRCA of class I and class II viruses and between best

369 represented genotypes within class II, we used a BMCMC approach as implemented in the

370 BEAST package (Drummond et al., 2012). The exponential growth model was the best fit to the

371 data (Griffiths and Tavare, 1994). Similar values were estimated using both GTR $+\Gamma_{4}$ and HKY + 
$372 \Gamma_{4}$ models (Table 2). Maximum clade credibility trees revealed that class I and class II APMV-1

373 have evolved from ancestors that existed between 1772 and 1782 (Table 2). Indeed, the long

374 period to the separation of class I and class II (approximately 234-244 years before the most

375 recent isolates included in this study) and the small differences in CAI of these two classes

376 provides evidence to suggest that the codon adaptation of APMV-1 is a slow process. As

377 expected, class II genotype VI emerged from common ancestors with genotype VII (and

378 genotypes XIII, XIV, XVII and XVIII) sometime between 1947 and 1951 (Table 2), that is in

379 agreement with previously reported estimates (Chong et al., 2013; Sonora et al., 2015). Despite

380 the differences in host of isolation for viruses of genotypes VI and VII, there are no large

381 differences in codon usage or host adaptation, as it would be expected from a slow process of

382 adaptation. Genotypes VII and VI separation is estimated to have occurred approximately 69-73

383 years ago. While genotype VI viruses are the cause of the third panzootic and maintained in

384 pigeons and doves, viruses of genotype VII belong to the fourth and fifth panzootics and have 385 been mainly isolated in chickens with occasional spillovers in other domestic and wild species

386 (Dimitrov et al., 2016). 


\section{Discussion}

While codon usage bias has been studied in other viruses of the Paramyxoviridae family

389 (Belalov and Lukashev, 2013), and in AMPV-1 (Kumar and Kumar, 2014; Wang et al., 2011),

390 this is the first study that shows that there are preferential codon usage within the two different

391 APMV-1 classes and that there are different codon usages among different genomic regions.

392 Preferential codon usage occurs across all domains of life, including viruses, in which this codon

393 usage preference enables viruses to increase or decrease replication rates (Behura and Severson,

394 2012; Moura et al., 2007). Previous studies have concentrated on codon usage bias in viral

395 families such as Orthomyxoviridae (Wong et al., 2010), Flaviviridae (Moratorio et al., 2013),

396 and Picornaviridae (Aragones et al., 2010) to show that viruses differ in their specificity of

397 codon usage depending on their host organism. Previously, codon usage bias was studied for 201

398 fusion genes (Kumar and Kumar, 2014) and 31 complete genomes (Wang et al., 2011) of

399 APMV-1 to determine if there were any host specific changes in codon usage; however, the

400 authors did not find any host adaptation in APMV-1. Here, using 1,341 fusion and 288 complete

401 genome sequences, we have extended those studies, and found differences between the two

402 AMPV-1 classes and among the different genomic regions of class I and class II.

The detection of codon usage adaptations in this manuscript may have been possible due

404 to the separation of sequences into recognized classes (41 to $46 \%$ nucleotide distance between

405 class II genotypes and class I), the higher number of codons utilized here, and the recognition

406 that the fusion gene may not best represent the mechanisms of codon usage adaptation in

407 APMV-1. The data indicates that codon usage bias for the fusion gene is distinct from other

408 genes and there is not a good correlation between class I and II fusion gene codon usage for this

409 gene. The observed low correlation when comparing the fusion gene RSCU between class I and 
410 class II sequences (Fig. 1B) indicates gene specific differences in codon usage bias, suggesting

411 the existence of different yet unknown types of evolutionary pressures affecting that bias in this

412 gene. The ENC vs CAI and GC3 data provides information that suggests selection plays a

413 predominant role in codon usage variability in class I fusion gene, whereas the codon usage

414 relations for class II fusion are more consistent with random mutations or with unknown

415 pressures. The fusion protein is a surface protein that has been shown to be affected by positive

416 selection and antibody selective pressures (Choi et al., 2010; Miller et al., 2009; Yusoff et al.,

417 1989); therefore, it is possible that fusion-specific selection may affect codon usage of this gene.

This study indicates that there are specific codon usage adaptations to yet unknown

419 pressures in the fusion and HN coding regions. Codon usage differences in three different

420 APMV-1 genomic regions appear to be related to expected transcriptional levels. In both classes,

421 the CAI of the highest expressed genes to Gallus gallus is higher than the CAI of the lowest

422 expressed genes (except for $\mathrm{F}$ and $\mathrm{HN}$ ), suggesting a possible role of codon usage on the control

423 of APMV-1 gene expression (Quax et al., 2015). The increased adaptation of the NC-PHO-M to

424 Gallus gallus is consistent with a higher use of host tRNAs, as this region would be translated

425 faster and more efficiently. Although different codons are preferentially used across the entire

426 genome and the positive correlation seen for the codon usage among the three genomic regions

427 indicate that class I and class II are varying together in the same magnitude and direction. Most

428 interesting are the deviations observed for the $\mathrm{F}$ and $\mathrm{HN}$ genes of class II viruses. A CAI gradient

429 from highly transcribed regions to low transcribed regions is clearly observed in viruses that are

430 mostly isolated from wild birds (class I) however the gradient in the CAI is interrupted in

431 viruses generally associated with chickens (class II) at the F and $\mathrm{HN}$ genes. This observation is

432 relevant because the evolution of $\mathrm{F}$ and $\mathrm{HN}$ genes of class II viruses is likely to be influenced by 
433 intensive vaccination in poultry. As the $\mathrm{F}$ and $\mathrm{HN}$ genes encode for surface proteins that contains

434 the sites of neutralization, it is possible that those two genes may evolve under different type of

435 pressures than the rest of the genome. It is possible that the discrepancies seen in codon usage

436 between the fusion protein and other genomic regions may be partially attributed to positive

437 selection caused by immune selective pressures on this gene because of its function as a surface

438 viral protein (Miller et al., 2009).

We infer that the fusion gene of class II viruses does not follow the same evolutionary

440 trend as the complete genome, which leads us to propose that any future analysis dealing with

441 codon usage bias should be done on both complete genomes and individual, essential genes.

442 Although all APMV-1s would have the same ancestral host, our data suggest that these two

443 classes of viruses have separated over two hundred years ago, thus it is likely that the different

444 classes may have adapted to replicate in different host species. As APMV-1s are reliant on the

445 avian host's translational machinery to synthesize the necessary proteins they need to replicate

446 (Walsh and Mohr, 2011), it would make sense that these viruses would evolve to use the host's

447 tRNA pool more efficiently (Pavon-Eternod et al., 2013). The differences in codon usage and the

448 differences in di-codon frequencies support this result. It is widely believed that virulent Class II

449 viruses (most of available sequences in GenBank) are maintained in vaccinated chicken, and

450 therefore, it would be expected that recent viruses would have significantly changed to adapt to

451 the host translational machinery. Surprisingly our codon adaption index data did not reveal any

452 significant increased adaptation on class II codon usage to chickens except for a minor increase

453 on the polymerase gene. Our results suggest that codon usage adaptation to chicken was not a

454 significant force in the rapid NDV evolution that has occurred in the last 40 years since 
vaccination started. It remains to be determined if other types of adaptations, such as adaptations to receptors or adaptation to the host translational machinery operate at the protein level.

To characterize the codon usage bias in the genotypes of class II viruses, we have separated the genotypes that are genetically related but clearly defined by the occurrence of significant genetic changes (above 10\% nucleotide differences between different genotypes) (Diel et al., 2012a; Dimitrov et al., 2016). Most viruses from class I are normally isolated from waterfowl, while viruses from class II have been isolated from a wide range of species. Some of those differences may be due to the existence of a maintenance cycle in other possible host organisms; with these hosts being different for the two classes and possibly different for the genotypes. However, a definitive identification of additional hosts is still lacking for some genotypes (e.g. genotype VII), and further investigation into the adaptation mechanisms of these viruses to their avian hosts need to be done.

Due to the high mobility of the viruses, the broad host ranges, and the slow rate of change, codon usage geographic or temporal changes are not likely to be easily detected. First, apart from a few exceptions in APMV-1, it is difficult to ascribe viruses to hosts. Spillovers between chickens and wild birds or among different wild bird species have been reported (Ayala et al., 2016; Cardenas Garcia et al., 2013; Dimitrov et al., 2016; Kim et al., 2007) and are likely to occur due to the wide host ranges of APMV-1s (Kaleta and Baldauf, 1988). Thus, it is possible to isolate identical or highly similar viruses from different host species, masking results on the adaptation to a host. A similar situation is likely when studying codon usage association with geographic or temporal factors. Our data and previous work suggest that efforts should be made to identify and exclude sequences from spillovers from any evolutionary studies (Ayala et al., 2016; Dimitrov et al., 2016), which may account for the failure of detection of patterns by others. 


\section{Acknowledgments}

479 We would like to thank Dr. Kateri Bertran, Dr. Dong-Hun Lee, and Dr. Jacob Spangler 480 for their useful comments on the manuscript, and Dr. Justin Bahl for his methodological support 481 in carrying tMRCA analyses. This work was supported by the BAA project [grant 482 \#685/FRCALL 12-6-2-0005]; and the USDA CRIS [grant 6612-32000-064-00D]. Mention of 483 trade names or commercial products in this publication is solely for the purpose of providing 484 specific information and does not imply recommendation or endorsement by the U.S. 485 Department of Agriculture. USDA is an equal opportunity provider and employer. 
Figure Legends:

487 Fig. 1. Class I and class II avian paramyxoviruses 1 display differences in codon usage within the fusion gene. A) The average relative synonymous codon usage (RSCU) value for each codon in the fusion gene ( $\mathrm{X}$ axis) was calculated using all available sequences (175 for CI and 1166 for $\mathrm{CII})$, and only the codon usage (Y axis) that was statistically different $(\mathrm{P}<0.01)$ between class I (blue) and class II (yellow) viruses is shown with standard error bars. Values can be found in Table S3; B) The average RSCU value for each codon in the fusion coding region of avian paramyxoviruses 1 (APMV-1) was used to assess the correlation of codon usage among 494 class I (X axis) and class II viruses (Y axis). $\mathrm{C}$ ) The ENC value (X axis) and GC3 value (Y axis) were calculated for the fusion gene of each APMV-1 sequence, and average values for all class I sequences $(\mathrm{n}=175)$ and the more prominent class II genotypes (104-GI, 18-GII, 87-GV, 261-

497 GVI, 474-GVII, 11-GX, 41-GXIII) are plotted on the graph. The 95\% confidence levels are indicated by the dotted lines; linear regression analysis provides a slope of $0.8086 \pm 0.01507$ with a significant $\mathrm{P}$ value $<0.0001$. D) The fusion gene $\mathrm{CAI}(\mathrm{X}$ axis) and ENC ( $\mathrm{Y}$ axis) values II sequences was created similarly to the plot in D.

Fig. 2. Avian paramyxoviruses 1 display differences in codon usage between the three genomic region (NC-PHO-M, F-HN, and POL) for class $\mathrm{I}(\mathrm{n}=29)$ and class II $(\mathrm{n}=259)$ viruses, was determined using Gallus gallus as a reference genome. The complete genome result is in black, NC-PHO-M is in blue, F-HN is in red, and POL is in green color. Standard error bars are shown and statistically significant differences between the same regions between classes are 
509 shown $(\mathrm{P}<0.01)$ with corresponding letters; B) The F-HN region CAI (X axis) and ENC (Y

510 axis) values were calculated and plotted for all class I sequences; C) The F-HN region CAI (X

511 axis) and ENC (Y axis) values were calculated and plotted for all class II sequences; D) For each

512 region, the ENC (X axis) and GC3 (Y axis) values were determined for each class I and II

513 sequence, and average values for each region from each class are plotted on the graph. NC-PHO-

514 M sequences are in blue, F-HN sequences are in red, and POL sequences are in green color.

516 Fig. 3. Differences and correlations in relative synonymous codon usage (RSCU) in the

517 complete genome between class I and class II avian paramyxoviruses 1. A) The average

518 RSCU value for each codon across the complete avian paramyxoviruses 1 (APMV-1) genome (X 519 axis) was calculated for all available sequences (29 for class I and 259 for class II), and only the 520 codon usage ( $\mathrm{Y}$ axis) that is statistically different $(\mathrm{P}<0.01)$ between class I (blue) and class II 521 (yellow) viruses are shown with standard error bars. Values can be found in Table S6; B) The 522 average RSCU value for each codon in the complete genome of APMV-1 was used to assess the 523 correlation of codon usage among class I ( $\mathrm{X}$ axis) and class II viruses ( $\mathrm{Y}$ axis); $\mathrm{C}$ ) The ENC (X 524 axis) and GC3 (Y axis) values were calculated for the complete genome of each APMV-1

525 sequence, and average values for all class I sequences $(n=29)$ and the more prominent class II 526 genotypes (28-GI, 5-GII, 17-GV, 73-GVI, 87-GVII, 8-GX, 14-GXIII) are plotted on the graph.

527 Linear regression analysis provides a slope of $0.8348 \pm 0.01521$ with a significant $\mathrm{P}$ value < $528 \quad 0.0001$. 
550 Afonso, C.L., Miller, P.J., 2013. Newcastle disease: progress and gaps in the development of 551 vaccines and diagnostic tools. Dev Biol (Basel) 135, 95-106.

552 Alexander, D.J., et al., 2012. The long view: a selective review of 40 years of Newcastle disease 553 research. Avian Pathol 41, 329-335.

554 Aragones, L., et al., 2010. Fine-tuning translation kinetics selection as the driving force of codon 555 usage bias in the hepatitis A virus capsid. PLoS Pathog 6, e1000797.

556 Ayala, A.J., et al., 2016. Presence of Vaccine-Derived Newcastle Disease Viruses in Wild Birds.

557 PLoS One 11, e0162484.

558 Bahir, I., et al., 2009. Viral adaptation to host: a proteome-based analysis of codon usage and 559 amino acid preferences. Mol Syst Biol 5, 311.

560 Behura, S.K., Severson, D.W., 2012. Comparative analysis of codon usage bias and codon 561 context patterns between dipteran and hymenopteran sequenced genomes. PLoS One 7, e43111.

562 Belalov, I.S., Lukashev, A.N., 2013. Causes and implications of codon usage bias in RNA 563 viruses. PLoS One 8, e56642.

564 Buchholz, C.J., et al., 1994. The carboxy-terminal domain of Sendai virus nucleocapsid protein 565 is involved in complex formation between phosphoprotein and nucleocapsid-like particles.

566 Virology 204, 770-776.

567 Cardenas Garcia, S., et al., 2013. Molecular epidemiology of Newcastle disease in Mexico and 568 the potential spillover of viruses from poultry into wild bird species. Appl Environ Microbiol 79, $569 \quad 4985-4992$.

570 Choi, K.S., et al., 2010. Antigenic and immunogenic investigation of the virulence motif of the 571 Newcastle disease virus fusion protein. J Vet Sci 11, 205-211. 
572 Chong, Y.L., et al., 2013. Successful establishment and global dispersal of genotype VI avian

573 paramyxovirus serotype 1 after cross species transmission. Infect Genet Evol 17, 260-268.

574 Coleman, J.R., et al., 2008. Virus attenuation by genome-scale changes in codon pair bias.

575 Science 320, 1784-1787.

576 Collins, P.L., et al., 1978. Transcription and translation of Newcastle disease virus mRNA's in

577 vitro. J Virol 28, 324-336.

578 Collins, P.L., et al., 1982. Coding assignments of the five smaller mRNAs of Newcastle disease $579 \quad$ virus. J Virol 43, 1024-1031.

580 Coronel, E.C., et al., 2001. Nucleocapsid incorporation into parainfluenza virus is regulated by 581 specific interaction with matrix protein. J Virol 75, 1117-1123.

582 Courtney, S.C., et al., 2013. Highly divergent virulent isolates of Newcastle disease virus from 583 the Dominican Republic are members of a new genotype that may have evolved unnoticed for 584 over 2 decades. J Clin Microbiol 51, 508-517.

585 Diel, D.G., et al., 2012a. Genetic diversity of avian paramyxovirus type 1: proposal for a unified 586 nomenclature and classification system of Newcastle disease virus genotypes. Infect Genet Evol $587 \quad 12,1770-1779$.

588 Diel, D.G., et al., 2012b. Complete genome and clinicopathological characterization of a virulent 589 Newcastle disease virus isolate from South America. J Clin Microbiol 50, 378-387.

590 Dimitrov, K.M., et al., 2016. Temporal, geographic, and host distribution of avian

591 paramyxovirus 1 (Newcastle disease virus). Infect Genet Evol 39, 22-34.

592 Dortmans, J.C., et al., 2010. Two genetically closely related pigeon paramyxovirus type 1

593 (PPMV-1) variants with identical velogenic fusion protein cleavage sites but with strongly 594 contrasting virulence. Vet Microbiol 143, 139-144. 
595 Drummond, A.J., et al., 2012. Bayesian phylogenetics with BEAUti and the BEAST 1.7. Mol 596 Biol Evol 29, 1969-1973.

597 Gao, Q., et al., 2008. Expression of transgenes from newcastle disease virus with a segmented 598 genome. J Virol 82, 2692-2698.

599 Gerdol, M., et al., 2015. Analysis of synonymous codon usage patterns in sixty-four different 600 bivalve species. PeerJ 3, e1520.

601 Glickman, R.L., et al., 1988. Quantitative basic residue requirements in the cleavage-activation 602 site of the fusion glycoprotein as a determinant of virulence for Newcastle disease virus. J Virol $603 \quad 62,354-356$.

604 Griffiths, R.C., Tavare, S., 1994. Sampling theory for neutral alleles in a varying environment. 605 Philos Trans R Soc Lond B Biol Sci 344, 403-410.

606 Hamaguchi, M., et al., 1983. Transcriptive complex of Newcastle disease virus. I. Both L and P 607 proteins are required to constitute an active complex. Virology 128, 105-117.

608 Huang, Z., et al., 2004. The hemagglutinin-neuraminidase protein of Newcastle disease virus 609 determines tropism and virulence. J Virol 78, 4176-4184.

610 Kaleta, E., Baldauf, C., 1988. Newcastle disease in free-living and pet birds, in: DJ, A. (Ed.),

611 Newcastle disease. Kluwer Academic Publishers, Boston, pp. 197-246.

612 Katoh, K., et al., 2002. MAFFT: a novel method for rapid multiple sequence alignment based on 613 fast Fourier transform. Nucleic Acids Res 30, 3059-3066.

614 Kim, L.M., et al., 2007. Phylogenetic diversity among low-virulence newcastle disease viruses 615 from waterfowl and shorebirds and comparison of genotype distributions to those of poultry616 origin isolates. J Virol 81, 12641-12653. 
617 Kim, S.H., et al., 2011. Roles of the fusion and hemagglutinin-neuraminidase proteins in

618 replication, tropism, and pathogenicity of avian paramyxoviruses. J Virol 85, 8582-8596.

619 Knight, R.D., et al., 2001. A simple model based on mutation and selection explains trends in

620 codon and amino-acid usage and GC composition within and across genomes. Genome Biol 2,

621 RESEARCH0010.

622 Kumar, C.S., Kumar, S., 2014. Species based synonymous codon usage in fusion protein gene of

623 Newcastle disease virus. PLoS One 9, e114754.

624 Mayo, M.A., 2002. Virus taxonomy - Houston 2002. Arch Virol 147, 1071-1076.

625 Miller, P.J., et al., 2009. Evolutionary dynamics of Newcastle disease virus. Virology 391, 64-

62672.

627 Moratorio, G., et al., 2013. A detailed comparative analysis on the overall codon usage patterns 628 in West Nile virus. Infect Genet Evol 14, 396-400.

629 Moura, G., et al., 2007. Large scale comparative codon-pair context analysis unveils general 630 rules that fine-tune evolution of mRNA primary structure. PLoS One 2, e847.

631 Nagai, Y., et al., 1976. Proteolytic cleavage of the viral glycoproteins and its significance for the 632 virulence of Newcastle disease virus. Virology 72, 494-508.

633 Pavon-Eternod, M., et al., 2013. Vaccinia and influenza A viruses select rather than adjust 634 tRNAs to optimize translation. Nucleic Acids Res 41, 1914-1921.

635 Quax, T.E., et al., 2015. Codon Bias as a Means to Fine-Tune Gene Expression. Mol Cell 59, 636 149-161.

637 Sharp, P.M., Li, W.H., 1986. An evolutionary perspective on synonymous codon usage in 638 unicellular organisms. J Mol Evol 24, 28-38. 
Sharp, P.M., Li, W.H., 1987. The codon Adaptation Index--a measure of directional synonymous

640 codon usage bias, and its potential applications. Nucleic Acids Res 15, 1281-1295.

641 Snoeck, C.J., et al., 2013. High genetic diversity of Newcastle disease virus in poultry in West

642 and Central Africa: cocirculation of genotype XIV and newly defined genotypes XVII and

643 XVIII. J Clin Microbiol 51, 2250-2260.

644 Sonora, M., et al., 2015. An evolutionary insight into Newcastle disease viruses isolated in 645 Antarctica. Arch Virol 160, 1893-1900.

646 Vicario, S., et al., 2007. Codon usage in twelve species of Drosophila. BMC Evol Biol 7, 226.

647 Walsh, D., Mohr, I., 2011. Viral subversion of the host protein synthesis machinery. Nat Rev

648 Microbiol 9, 860-875.

649 Wang, M., et al., 2011. Analysis of codon usage in Newcastle disease virus. Virus Genes 42, $650 \quad 245-253$.

651 Wilde, A., et al., 1986. Identification of the sequence content of four polycistronic transcripts 652 synthesized in Newcastle disease virus infected cells. Virus Res 5, 77-95.

653 Wong, E.H., et al., 2010. Codon usage bias and the evolution of influenza A viruses. Codon 654 Usage Biases of Influenza Virus. BMC Evol Biol 10, 253.

655 Wright, F., 1990. The 'effective number of codons' used in a gene. Gene 87, 23-29.

656 Xia, X.H., 2013. DAMBE5: A Comprehensive Software Package for Data Analysis in Molecular 657 Biology and Evolution. Molecular Biology and Evolution 30, 1720-1728.

658 Yusoff, K., et al., 1989. Location of neutralizing epitopes on the fusion protein of Newcastle 659 disease virus strain Beaudette C. J Gen Virol 70 ( Pt 11), 3105-3109.

Table 1. Relative synonymous codon usage values of the three genomic regions in class I and class II avian paramyxoviruses 1. 


\begin{tabular}{|c|c|c|c|c|c|c|c|}
\hline \multirow[b]{2}{*}{ Codon } & \multirow[b]{2}{*}{ AA } & \multicolumn{2}{|c|}{ NC-PHO-M } & \multicolumn{2}{|c|}{$\mathrm{F}-\mathrm{HN}$} & \multicolumn{2}{|c|}{ POL } \\
\hline & & Class I & Class II & Class I & Class II & Class I & Class II \\
\hline GCU & $A$ & 0.87 & 0.89 & 0.84 & 0.87 & 0.83 & 0.98 \\
\hline GCG & $A$ & 0.45 & 0.54 & 0.42 & 0.48 & 0.37 & 0.49 \\
\hline GCC & $A$ & 1.09 & 1 & 0.99 & 0.91 & 0.94 & 0.7 \\
\hline GCA & $A$ & 1.6 & 1.57 & 1.75 & 1.74 & 1.85 & 1.83 \\
\hline UGU & $C$ & 0.82 & 0.63 & 1.09 & 1.08 & 1.08 & 1.06 \\
\hline UGC & $C$ & 1.18 & 1.37 & 0.91 & 0.92 & 0.92 & 0.94 \\
\hline GAU & $\mathrm{D}$ & 0.89 & 0.91 & 1.15 & 1.08 & 1.07 & 1.16 \\
\hline GAC & $D$ & 1.11 & 1.09 & 0.85 & 0.92 & 0.93 & 0.84 \\
\hline GAG & $E$ & 1.16 & 1.25 & 1.2 & 0.79 & 1.1 & 1.15 \\
\hline GAA & $\mathrm{E}$ & 0.84 & 0.75 & 0.8 & 1.21 & 0.9 & 0.85 \\
\hline UUU & $\mathrm{F}$ & 0.72 & 0.96 & 0.82 & 0.96 & 0.83 & 0.87 \\
\hline UUC & $\mathrm{F}$ & 1.28 & 1.04 & 1.18 & 1.04 & 1.17 & 1.13 \\
\hline GGU & G & 0.76 & 0.69 & 0.76 & 0.95 & 0.75 & 0.9 \\
\hline GGG & G & 1.31 & 1.3 & 1.53 & 1.42 & 1.53 & 1.2 \\
\hline GGC & G & 0.78 & 0.75 & 0.6 & 0.8 & 0.79 & 0.72 \\
\hline GGA & G & 1.14 & 1.26 & 1.11 & 0.83 & 0.94 & 1.18 \\
\hline CAC & $\mathrm{H}$ & 1.11 & 0.78 & 0.68 & 0.87 & 0.83 & 0.83 \\
\hline CAU & $\mathrm{H}$ & 0.89 & 1.22 & 1.32 & 1.13 & 1.17 & 1.17 \\
\hline AUU & 1 & 1.02 & 0.81 & 0.78 & 0.68 & 0.99 & 1.1 \\
\hline$A \cup A$ & 1 & 0.78 & 0.66 & 1.16 & 1.26 & 0.94 & 0.92 \\
\hline$A \cup C$ & 1 & 1.2 & 1.53 & 1.05 & 1.07 & 1.07 & 0.98 \\
\hline AAA & K & 0.85 & 0.8 & 0.86 & 0.86 & 0.88 & 0.94 \\
\hline AAG & K & 1.15 & 1.2 & 1.14 & 1.14 & 1.12 & 1.06 \\
\hline CUA & $\mathrm{L}$ & 0.83 & 0.71 & 1.01 & 0.93 & 1.32 & 1.08 \\
\hline CUC & $\mathrm{L}$ & 1.22 & 1.38 & 0.97 & 1 & 1.01 & 0.99 \\
\hline CUG & $\mathrm{L}$ & 0.92 & 0.9 & 1.18 & 1 & 0.91 & 1.04 \\
\hline CUU & $\mathrm{L}$ & 1.03 & 1.02 & 0.84 & 1.07 & 0.76 & 0.88 \\
\hline UUA & $\mathrm{L}$ & 0.78 & 1.17 & 0.9 & 1.24 & 0.87 & 1.1 \\
\hline UUG & $\mathrm{L}$ & 1.22 & 0.83 & 1.1 & 0.76 & 1.13 & 0.9 \\
\hline$A A C$ & $\mathrm{~N}$ & 1.22 & 0.89 & 0.78 & 0.74 & 0.95 & 0.82 \\
\hline AAU & $\mathrm{N}$ & 0.78 & 1.11 & 1.22 & 1.26 & 1.05 & 1.18 \\
\hline CCA & $P$ & 1.42 & 1.15 & 1.35 & 0.89 & 1.44 & 1.53 \\
\hline $\mathrm{CCC}$ & $P$ & 0.95 & 0.74 & 0.86 & 1.09 & 0.46 & 0.66 \\
\hline $\mathrm{CCU}$ & $P$ & 0.88 & 1.24 & 1.22 & 1.36 & 0.98 & 1.23 \\
\hline CCG & $\mathrm{P}$ & 0.76 & 0.87 & 0.56 & 0.66 & 1.12 & 0.58 \\
\hline CAA & $\mathrm{Q}$ & 0.79 & 0.93 & 0.84 & 1.23 & 0.99 & 0.92 \\
\hline CAG & $Q$ & 1.21 & 1.07 & 1.16 & 0.77 & 1.01 & 1.08 \\
\hline $\mathrm{AGA}$ & $\mathrm{R}$ & 0.92 & 1.01 & 0.79 & 1.03 & 0.85 & 1.01 \\
\hline AGG & $\mathrm{R}$ & 1.08 & 0.99 & 1.21 & 0.97 & 1.15 & 0.99 \\
\hline CGA & $\mathrm{R}$ & 0.33 & 0.96 & 1.72 & 0.8 & 1.49 & 1.23 \\
\hline CGC & $\mathrm{R}$ & 1.14 & 0.9 & 1.12 & 1.11 & 1.02 & 0.5 \\
\hline CGG & $\mathrm{R}$ & 1.54 & 1.34 & 0.65 & 1.32 & 0.85 & 1.18 \\
\hline
\end{tabular}




\begin{tabular}{lccccccc} 
CGU & $\mathrm{R}$ & $\mathbf{0 . 9 9}$ & $\mathbf{0 . 8 1}$ & $\mathbf{0 . 5}$ & $\mathbf{0 . 7 7}$ & $\mathbf{0 . 6 4}$ & $\mathbf{1 . 0 8}$ \\
AGC & $\mathrm{S}$ & 1.25 & 1.23 & 0.96 & 1.06 & 1.25 & 1.03 \\
AGU & $\mathrm{S}$ & 0.75 & 0.77 & 1.04 & 0.94 & 0.75 & 0.97 \\
UCA & $\mathrm{S}$ & $\mathbf{1 . 6 7}$ & $\mathbf{1 . 0 7}$ & $\mathbf{1 . 1 1}$ & $\mathbf{1 . 4 5}$ & $\mathbf{1 . 5 6}$ & $\mathbf{1 . 4 5}$ \\
UCC & $\mathrm{S}$ & $\mathbf{0 . 7 9}$ & $\mathbf{1 . 0 2}$ & $\mathbf{0 . 9 7}$ & $\mathbf{0 . 7 6}$ & $\mathbf{0 . 6 4}$ & $\mathbf{0 . 9 1}$ \\
UCG & $\mathrm{S}$ & 0.43 & 0.47 & 0.52 & 0.36 & 0.42 & 0.36 \\
UCU & $\mathrm{S}$ & $\mathbf{1 . 1 1}$ & $\mathbf{1 . 4 3}$ & 1.41 & 1.44 & $\mathbf{1 . 3 9}$ & $\mathbf{1 . 2 8}$ \\
ACC & $\mathrm{T}$ & $\mathbf{1 . 1 1}$ & $\mathbf{1 . 2 5}$ & 0.97 & 1 & $\mathbf{0 . 9 4}$ & $\mathbf{0 . 7 9}$ \\
ACA & $\mathrm{T}$ & $\mathbf{1 . 4 5}$ & $\mathbf{1 . 2}$ & 1.55 & 1.5 & 1.55 & 1.61 \\
ACG & $\mathrm{T}$ & $\mathbf{0 . 3 6}$ & $\mathbf{0 . 2 4}$ & $\mathbf{0 . 4 2}$ & $\mathbf{0 . 3 2}$ & $\mathbf{0 . 6 3}$ & $\mathbf{0 . 3 9}$ \\
ACU & $\mathrm{T}$ & $\mathbf{1 . 0 8}$ & $\mathbf{1 . 3}$ & $\mathbf{1 . 0 7}$ & $\mathbf{1 . 1 8}$ & $\mathbf{0 . 8 8}$ & $\mathbf{1 . 2 1}$ \\
GUU & $\mathrm{V}$ & $\mathbf{0 . 4 2}$ & $\mathbf{0 . 7 5}$ & $\mathbf{0 . 5 1}$ & $\mathbf{0 . 8 4}$ & 0.56 & 0.63 \\
GUG & $\mathrm{V}$ & $\mathbf{1 . 8 7}$ & $\mathbf{1 . 2 2}$ & $\mathbf{1 . 3}$ & $\mathbf{0 . 9 7}$ & 1.14 & 1.11 \\
GUC & $\mathrm{V}$ & $\mathbf{0 . 9 6}$ & $\mathbf{1 . 1 9}$ & $\mathbf{0 . 7 6}$ & $\mathbf{1 . 1 1}$ & $\mathbf{1 . 1 8}$ & $\mathbf{1 . 1}$ \\
GUA & $\mathrm{V}$ & 0.75 & 0.84 & 1.43 & 1.09 & 1.12 & 1.16 \\
UAC & $\mathrm{Y}$ & $\mathbf{1 . 2 9}$ & $\mathbf{1 . 0 9}$ & 0.96 & 0.91 & $\mathbf{0 . 9}$ & $\mathbf{1 . 0 2}$ \\
UAU & $\mathrm{Y}$ & $\mathbf{0 . 7 1}$ & $\mathbf{0 . 9 1}$ & 1.04 & 1.09 & $\mathbf{1 . 1}$ & $\mathbf{0 . 9 8}$ \\
\hline
\end{tabular}

* Codons in bold font indicate the codon usage that is statistically different between class I and class II

*Values in color indicate the codon usage that is statistically different between class I and class II only in that specific region (NC-PHO-M = blue; F-HN = Red; POL = Green)

*NC-PHO-M: Class I = 36,163 codons; Class II = 322,973

$*$ F-HN: Class I $=32,596$; Class II $=291,116$

$*$ POL: Class I $=63,916$; Class II $=570,836$ 
Table 2. Bayesian estimates of demographic parameters as inferred from Bayesian Markov Chain Monte Carlo analyses of 155 APMV-1 polymerase gene sequences using relaxed lognormal molecular clock.

\begin{tabular}{|c|c|c|c|c|c|}
\hline Group & Model & $\begin{array}{c}\text { Demographic } \\
\text { model }\end{array}$ & $\begin{array}{c}\text { Date } \\
\text { (years) }\end{array}$ & $\begin{array}{l}\text { Time to } \mathrm{MRCA}^{\mathrm{a}} \\
\text { In years }\end{array}$ & $\begin{array}{c}\text { Time to MRCA } \\
\text { In years }\left(95 \% \mathrm{HPD}^{\mathrm{b}}\right)\end{array}$ \\
\hline \multirow{2}{*}{ Class I and class II } & $\mathrm{GTR}+\Gamma_{4}$ & Exponential & 1782 & 234 & $191 / 259$ \\
\hline & $\mathrm{HKY}+\mathrm{\Gamma}_{4}$ & Exponential & 1772 & 244 & $176 / 336$ \\
\hline Genotype VI and & $\mathrm{GTR}+\Gamma_{4}$ & Exponential & 1945 & 71 & $63 / 73$ \\
\hline Genotype VII & $\mathrm{HKY}+\mathrm{\Gamma}_{4}$ & Exponential & 1949 & 67 & $58 / 70$ \\
\hline
\end{tabular}

${ }^{\mathrm{a}} \mathrm{MRCA}=$ most recent common ancestor

${ }^{b} \mathrm{HPD}=$ highest posterior density 
A.

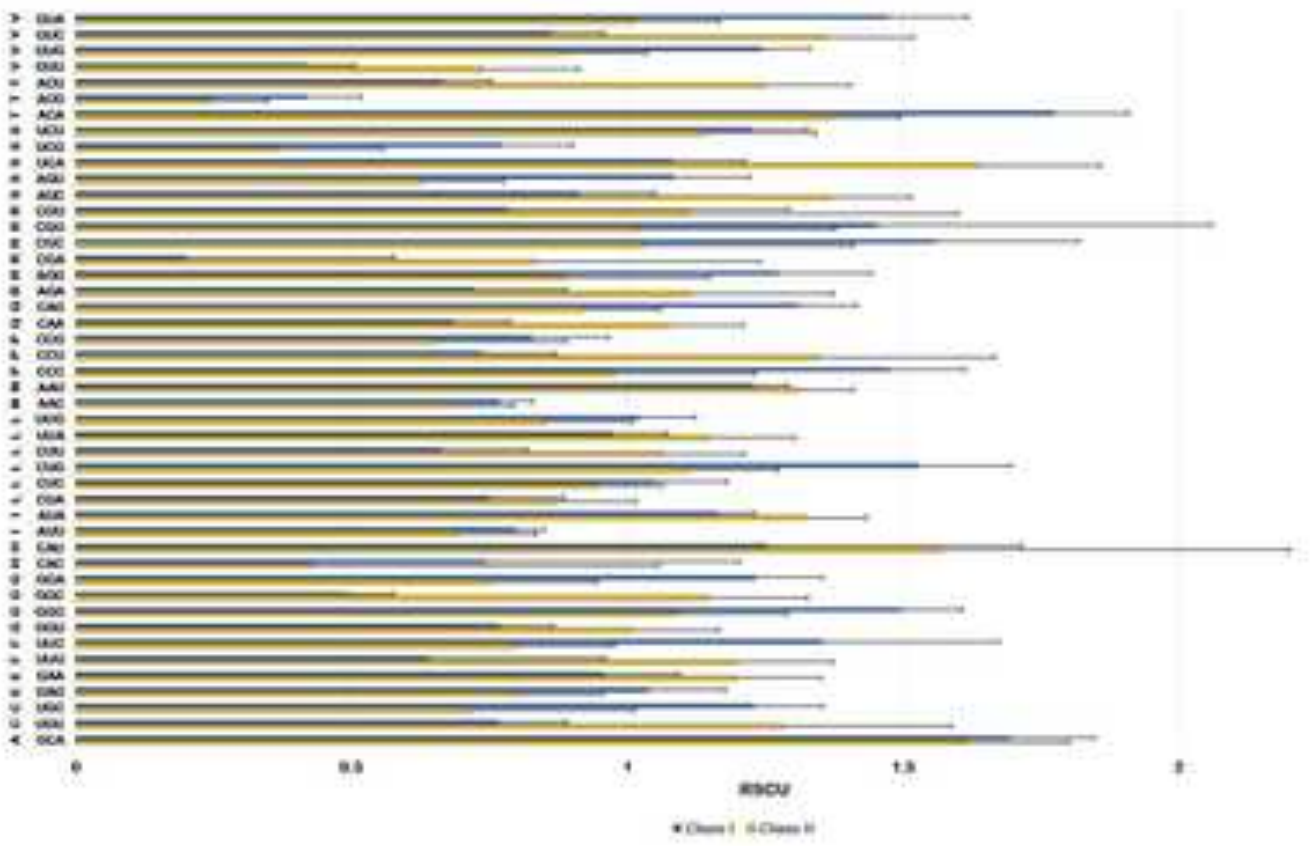

B.

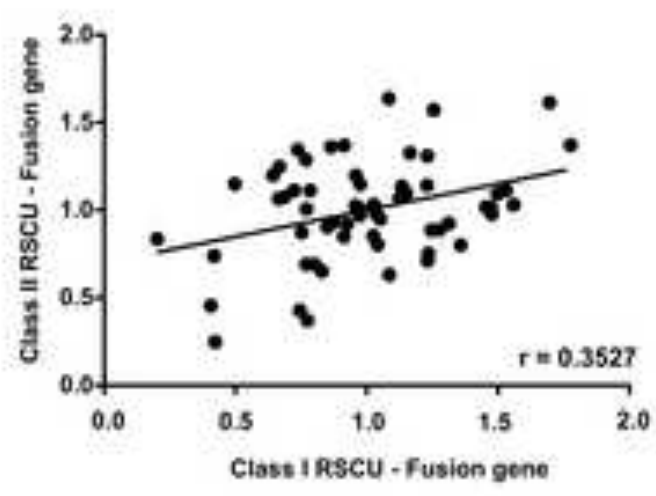

D.

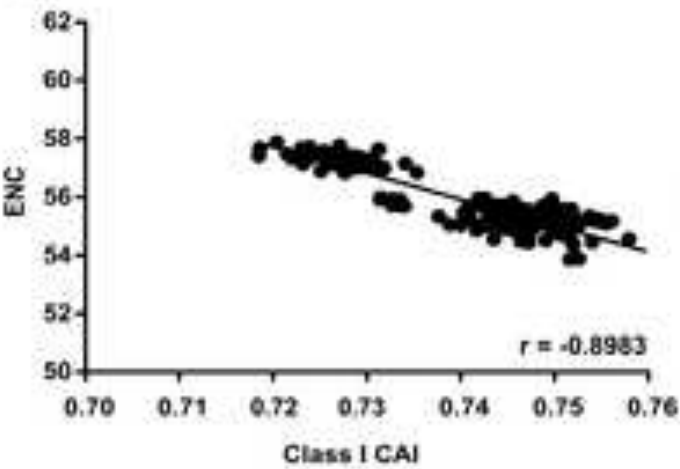
Class ICA
E. c.
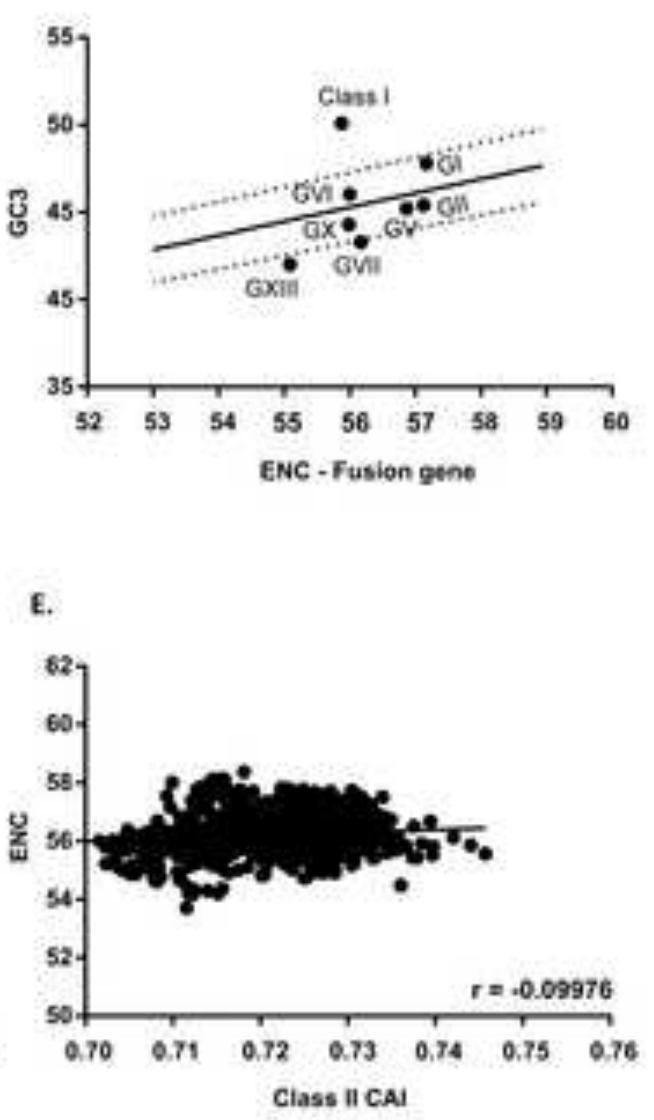

\section{Figure(s)}

A. 
A.

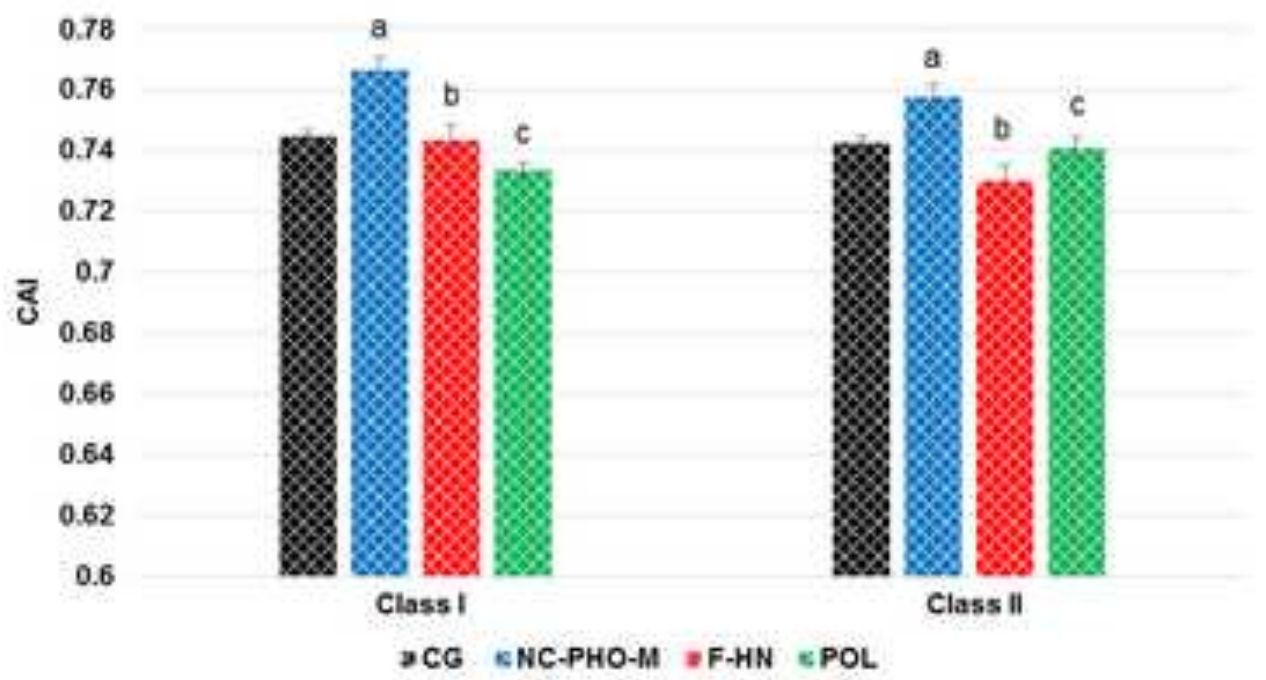

B.

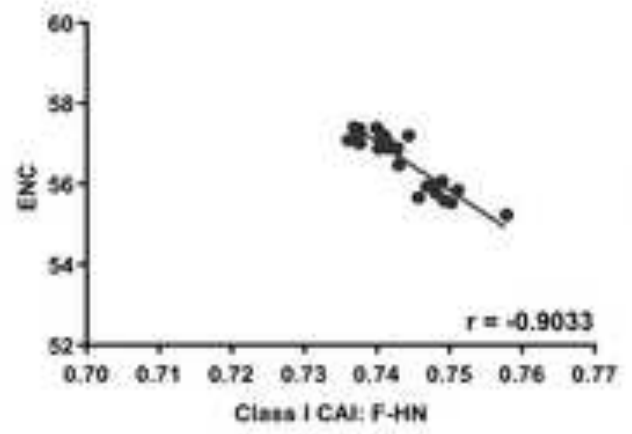

c.

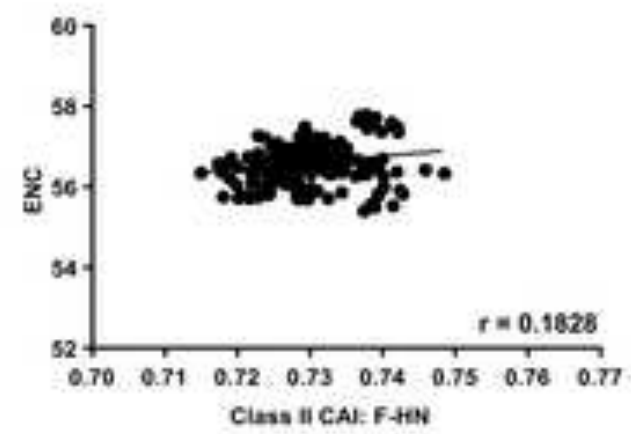

D.

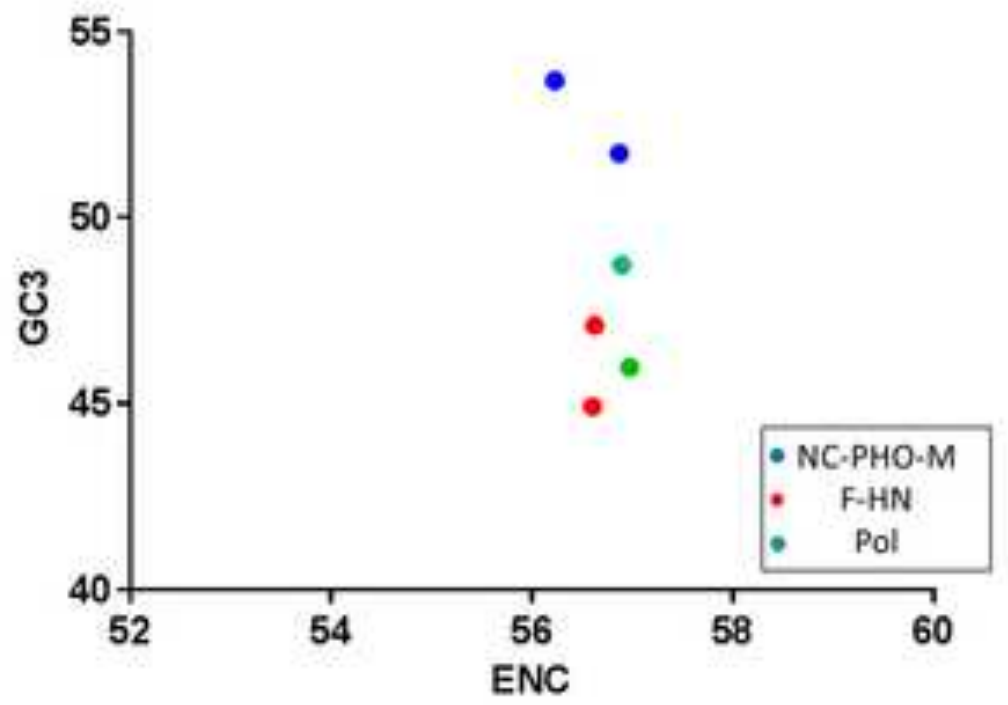


A.

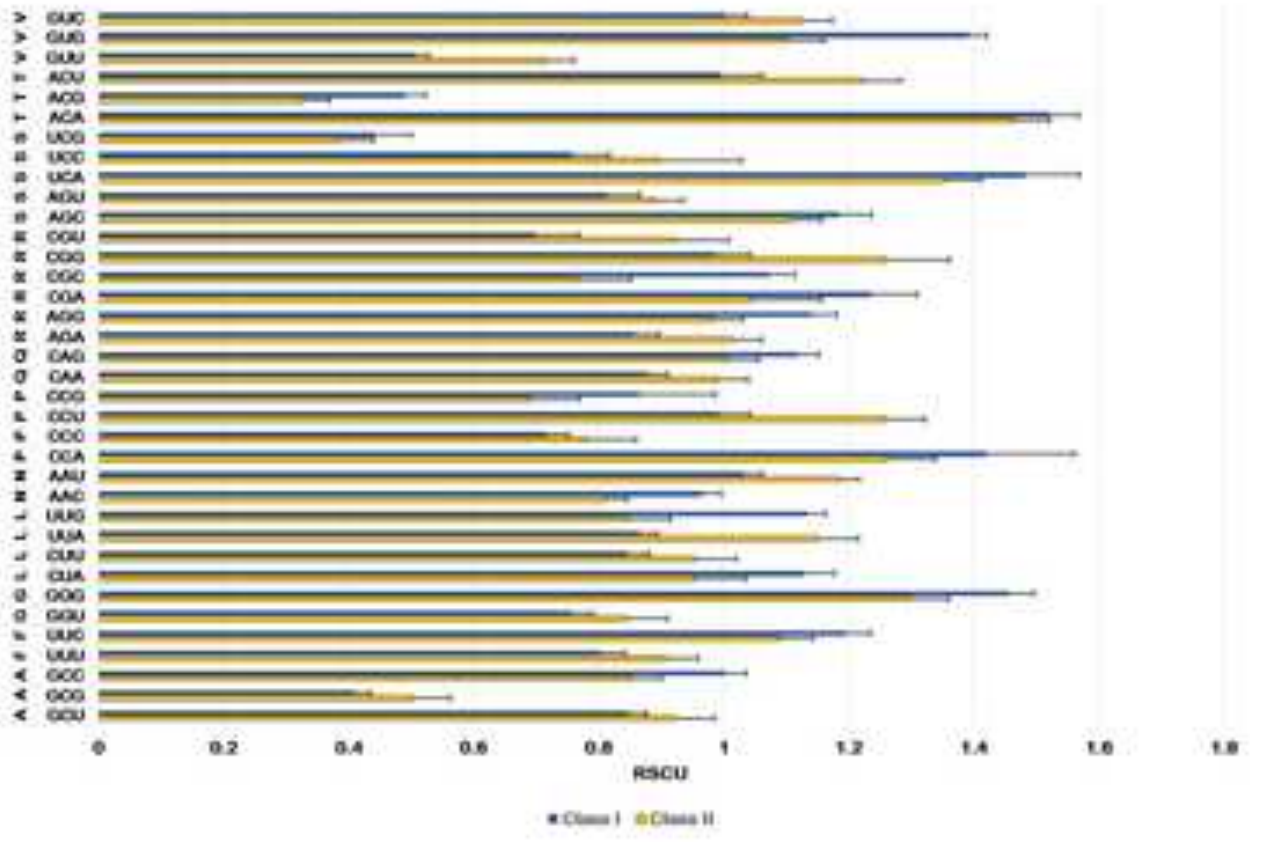

B.

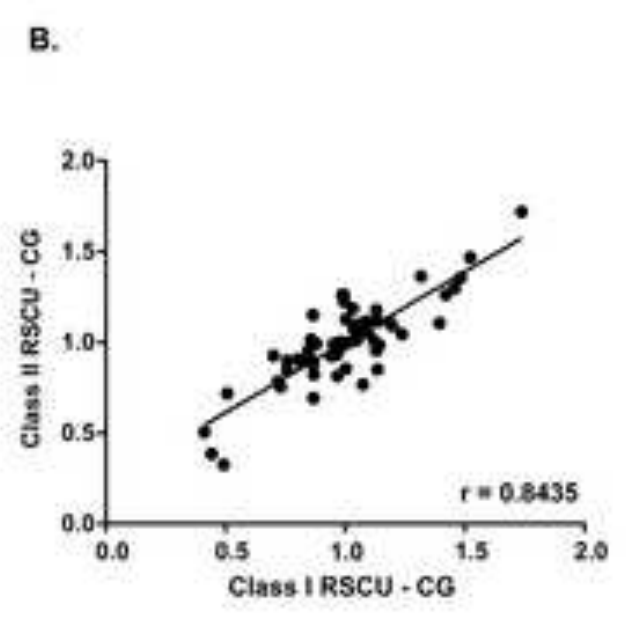
Class I RSCU - CG
. c.

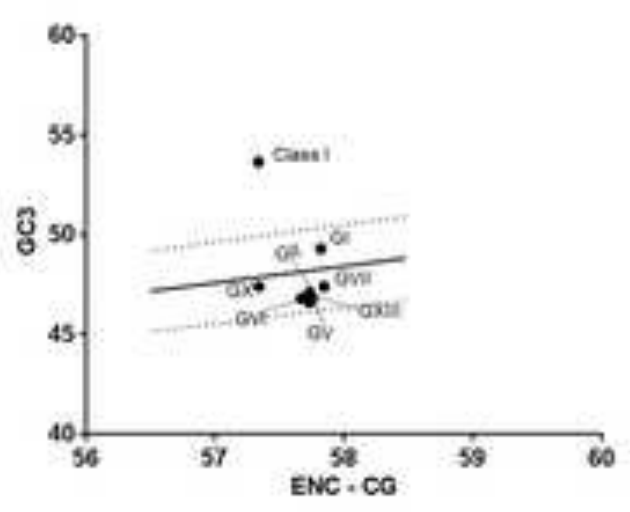

Figure(s) 3

-clan I bCles II

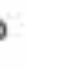

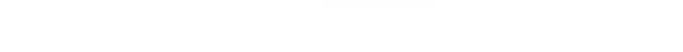

